\title{
Finite-amplitude interfacial waves in the presence of a current
}

\author{
By PHILIP G. SAFFMAN AND HENRY C. YUEN† \\ Applied Mathematics, California Institute of Technology, Pasadena, California 91125
}

(Received 16 November 1981 and in revised form 16 April 1982)

Solutions for interfacial waves of permanent form in the presence of a current were obtained for small-to-moderate wave amplitudes. A weakly nonlinear approximation was used to give simple analytical solutions to second order in wave height. Numerical methods were used to obtain solutions for larger wave amplitudes; details are reported for a number of selected cases. A special class of finite-amplitude solutions, closely related to the well-known Stokes surface waves, were identified. Factors limiting the existence of steady solutions are examined.

\section{Introduction}

We consider gravity waves at the interface between two uniform unbounded fluids of different densities in the presence of a relative horizontal velocity $U$. The fluids are supposed to be incompressible and inviseid, and the motion is assumed to be irrotational. We present here the properties of finite-amplitude periodic waves with wavelength $L$, which propagate steadily without change of shape with speed $C$ in the direction of $U$. We shall call these waves two-dimensional. For the purpose of calculating steady waves, there is no loss of generality in taking $C$ parallel to $U$, as an arbitrary constant transverse velocity may be linearly superposed on any two-dimensional steady waves (the stability properties would, however, be affected). It is convenient to reduce the wave propagation to rest by moving with the wave. The flow is then independent of time, and is sketched in figure 1. The speed of the wave is measured relative to the flow of the lower fluid at infinite depth.

We shall denote properties of the upper fluid by primed (') quantities, and those of the lower fluid by unprimed quantities. Without loss of generality, we can take the wavelength $L=2 \pi$, the gravitational acceleration $g=1$, and the lower density $\rho=1$. The mathematical problem is to determine the $x$-periodic velocity potentials and stream functions $\phi, \phi^{\prime}, \psi, \psi^{\prime}$, which satisfy Laplace's equation and are harmonic conjugate pairs, so that at the unknown interface $y=Y(x)$,

$$
\begin{aligned}
\psi[x, Y(x)] & =0 \\
\psi^{\prime}[x, Y(x)] & =0 \\
-p=b+Y(x)+\frac{1}{2}(\nabla \phi)^{2} & =r_{\mathrm{B}} Y(x)+\frac{1}{2} r\left(\nabla \phi^{\prime}\right)^{2}
\end{aligned}
$$

where $r=\rho^{\prime} / \rho$. In general, $r_{\mathrm{B}}=r$, but we allow for the possibility of Boussinesq waves to be obtained by setting $r=1$ and $r_{\mathrm{B}}=0$.

+ Present address: Fluid Mechanics Department, TRW Space and Technology Group One Space Park, Redondo Beach, CA 90278. 


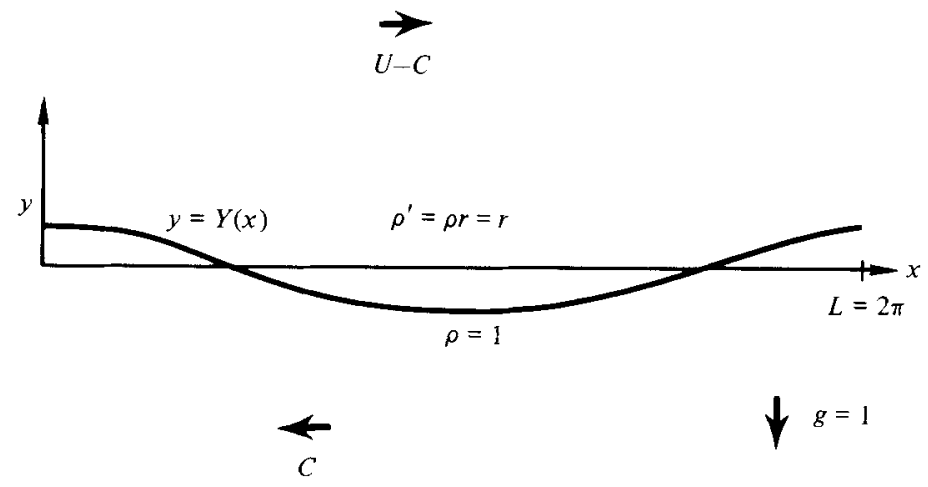

FTGURE 1. Sketch of flow configuration.

Surface tension at the interface is neglected. The origin of $Y$ is fixed by the requirement that the mean elevation is zero, i.e.

$$
\bar{y}=\frac{1}{2 \pi} \int_{0}^{2 \pi} Y(x) d x=0 .
$$

The conditions that the flow is uniform far below and above the interface give

$$
\begin{gathered}
\phi \sim-C x \text { as } y \rightarrow-\infty, \\
\phi^{\prime} \sim(U-C) x \text { as } y \rightarrow+\infty .
\end{gathered}
$$

Setting the right-hand sides of (1.1) and (1.2) equal to zero fixed the arbitrary constants in the stream functions $\psi$ and $\psi^{\prime}$ and, likewise, the pressure was completely determined by (1.3), where the Bernoulli constant was set to zero in the upper fluid and was given the value $b$ in the lower fluid. The problem specified by (1.1)-(1.6) is then free from arbitrary constants and the wave is determined by its trough-to-crest height $H$.

It is expected that isolated families of solutions exist in connected regions in the $(H, r, U)$-space, although, to our knowledge, this property has not been proved rigorously.

For $r=0$ (surface gravity waves), the literature on waves of permanent form is extensive. When $r>0$, the study of infinitesimal waves $(H \ll 1)$ is classical (see Lamb $1932, \S 231$ et seq.). For interfacial waves of permanent form with $U=0$, Tsuji \& Nagata (1973) earried out a Stokes-type expansion in wave amplitude to fifth order. Holyer (1979) extended these calculations for various values of $r$ to finite amplitude, using the computer to do the algebra, and 'summed' the series using Padé approximants. To our knowledge, no work has been reported on finite-amplitude interfacial waves with non-zero current.

In the presence of a current, the waves are functions of three parameters: $H, r$ and $U$. For small $H$, simple algebraic expressions have been obtained for the leading-order nonlinear corrections. For larger values of $H$, only numerical results are obtained. We shall present representative results for selected values of $H, r$ and $U$, and pay attention to two distinct factors that limit the existence of steady waves.

The first factor is what we term a 'dynamical limit'. This limit is encountered when $U$ is increased with $H$ fixed. For $H$ very smali, this is associated with the existence of the well-known Kelvin-Helmholtz instability, which may be interpreted as the 
non-existence of steady linear waves of a given wavelength when $U$ is sufficiently large. For finite-amplitude waves, this limit is identified by the system of equations describing steady waves becoming singular and solutions failing to exist, even though the 'limiting wave' profile is smooth and exhibits no unphysical properties.

The second factor is what we term a 'geometrical limit', at which the wave profiles become unphysical, even though the mathematical formulation may remain wellbehaved. This occurs for fixed $U$ and increasing $H$. Examples of this phenomenon are found in pure capillary and capillary-gravity waves for which the wave profile crosses itself at a critical value of $H$ (Crapper 1957; Chen \& Saffman 1980a).

In $\S 2$, we give the mathematical formulation of the problem. In $\$ 3$, we give results for weakly nonlinear waves based on the averaged variational principle. The numerical methods are described in $\$ 4$, and the main results are presented in $\$ 5$. In $\$ 6$, we exhibit a special class of exact solutions related to the Stokes surface waves. Some remarks regarding the limits of solutions are given in $\$ 7$.

The stability of the steady waves to two- and three-dimensional disturbances and the existence of three-dimensional bifurcations will also be reported on in subsequent papers.

\section{Mathematical formulation in physical space}

We write the interface as a Fourier series

$$
Y(x)=\sum_{1}^{\infty} A_{n} \cos n x
$$

The crest is taken to be at $x=0$ and the wave is assumed to be symmetrical about the crest and the trough which is at $x=\pi$. (The existence of non-symmetrical waves is an open question.) The velocity potential and stream functions below and above the interface are written as

$$
\begin{gathered}
\phi=-C x+\sum_{1}^{\infty} B_{n} \sin n x \exp \left[n\left(y-y_{\mathrm{c}}\right)\right] \\
\psi=-C y+B_{0}+\sum_{1}^{\infty} B_{n} \cos n x \exp \left[n\left(y-y_{\mathrm{c}}\right)\right] \\
\phi^{\prime}=(U-C) x+\sum_{1}^{\infty} B_{n}^{\prime} \sin n x \exp \left[-n\left(y-y_{\mathrm{t}}\right)\right] \\
\psi^{\prime}=(U-C) y+B_{0}^{\prime}+\sum_{1}^{\infty} B_{n}^{\prime} \cos n x \exp \left[-n\left(y-y_{\mathrm{t}}\right)\right] \\
y_{\mathrm{c}}=\sum_{1}^{\infty} A_{n}, \quad y_{\mathrm{t}}=\sum_{1}^{\infty}(-1)^{n} A_{n}
\end{gathered}
$$

Here

are the levels of the crest and the trough, related by

$$
y_{\mathrm{c}}-y_{\mathrm{t}}=H \text {. }
$$

The unknown coefficients $A_{n}, B_{n}, B_{n}^{\prime}$, together with the unknown Bernoulli coefficient $b$ and the wave speed $C$, are determined by substitution into (1.1)-(1.3) and equating coefficients of $\cos n x$. This gives an infinite set of equations in as many unknowns. The first few terms can be found by algebraic manipulation, which quickly becomes impossible as the number of terms increases. To obtain a substantial number of terms, either the computer must be used to perform the algebra (as in Holyer 1979), or alternative numerical methods must be employed. In $\$ 4$ we shall describe two 
different numerical approaches that we have taken for calculating the results presented here.

Certain integral properties of the wave are of interest. The kinetic-energy density relative to the undisturbed flow is

$$
T^{\prime}=-\frac{1}{2} B_{0} C+\frac{1}{2} r B_{0}^{\prime}(C+U),
$$

and the potential-energy density is

$$
V=\frac{1}{4}\left(1-r_{\mathrm{B}}\right) \sum_{1}^{\infty} A_{n}^{2}
$$

The momentum flux (or radiation stress) $S_{x x}$ relative to the undisturbed flow is given by

$$
S_{x x}=4 T-3 V-2 r U B_{0}^{\prime} .
$$

The other integral quantities, such as action, momentum and energy flux, can be derived from these using the Lagrangian formulation (see (3.10) below). The derivation of $(2.8)$ and $(2.10)$ is postponed to the appendix, where it is also shown that the Bernoulli constant $b$ is related to $C$ by the relation

$$
b=\frac{1}{2} r(U-C)^{2}-\frac{1}{2} C^{2} .
$$

This equation is used to check the accuracy of the numerical solutions.

\section{Weakly nonlinear approximations}

The properties of weakly nonlinear steady waves may be obtained by using the Stokes expansion (Stokes 1847), as done by Tsuji \& Nagata (1973) for the case of interfacial waves with no current. However, the algebra is greatly reduced when one employs the averaged variational principle (see Whitham 1974, §16.6). For irrotational interfacial waves, with a current $U$, the averaged Lagrangian is given by

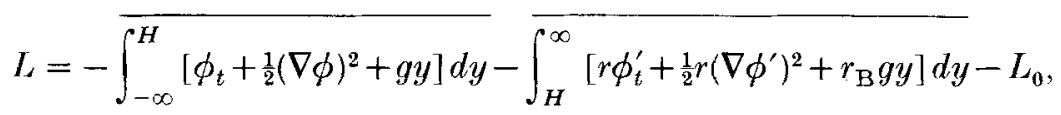

where the overbar denotes a time or space average, and

$$
L_{0}=-\int_{-\infty}^{0} g y d y-\int_{0}^{\infty}\left(\frac{1}{2} r U^{2}+r_{\mathrm{B}} g y\right) d y
$$

is the Lagrangian density of the undisturbed flow. A convergent value for $L$ is obtained by taking the limits together in all integrals, so that the infinity associated with the infinite depth is cancelled. In fact, $L$ can be thought of as the perturbation Lagrangian density with reference to the uniform flow. Note that, in the present section, the frame of reference is fixed relative to the fluid at infinite depth.

Following Whitham (1974), we substitute the leading-order terms for the wave profile and the velocity potentials

$$
\begin{gathered}
Y(x)=\frac{1}{2} H \cos (k x-\omega t)+a_{2} \cos 2(k x-\omega t), \\
\phi(x, y)=b_{1} \sin (k x-\omega t) e^{k y}+b_{2} \sin 2(k x-\omega t) e^{2 k y}, \\
\phi^{\prime}(x, y)=U x+b_{1}^{\prime} \sin (k x-\omega t) e^{-k y}+b_{2}^{\prime} \sin 2(k x-\omega t) e^{-2 k y} .
\end{gathered}
$$

We anticipate that $b_{1}$ and $b_{1}^{\prime}$ are $O(H)$ and that $a_{2}, b_{2}$ and $b_{2}^{\prime}$ are $O\left(H^{2}\right)$. We substitute into (3.1), evaluate $L$, solve for and eliminate $b_{1}, b_{2}, b_{1}^{\prime}$, and $b_{2}^{\prime}$ by the equations

$$
\frac{\partial L}{\partial b_{1}}=\frac{\partial L}{\partial b_{1}^{\prime}}=\frac{\partial L}{\partial b_{2}}=\frac{\partial L}{\partial b_{2}^{\prime}}=0
$$


and after some algebra obtain

$$
\begin{aligned}
L=\frac{1}{4} g\left(r_{\mathrm{B}}-1\right)\left(\frac{1}{4} H^{2}+a_{2}^{2}\right)+\frac{H^{2}}{16 k}\left[\omega^{2}+r(U k-\omega)^{2}\right]-\frac{a_{2}^{2}}{2 k}\left[\omega^{2}+r(U k-\omega)^{2}\right] \\
-\frac{1}{256} k H^{4}\left[\omega^{2}+r(U k-\omega)^{2}\right]-\frac{1}{16} H^{2} a_{2}\left[\omega^{2}-r(U k-\omega)^{2}\right]+O\left(H^{6}\right) .
\end{aligned}
$$

The value of $a_{2}$ is found from $\partial L / \partial a_{2}=0$ to be

$$
a_{2}=\frac{H^{2}}{8 g\left(1-r_{\mathrm{B}}\right)}\left[C^{2}-r(U-C)^{2}\right]+O\left(H^{4}\right)
$$

where $C=\omega / k$ is the linear phase speed. The dispersion relation for the weakly nonlinear wave then follows from $\partial L / \partial H=0$ :

$$
C^{2}+r(U-C)^{2}=\frac{g}{k}\left(1-r_{\mathrm{B}}\right)\left[1+\frac{1}{8} H^{2}\left(2 \frac{k C^{2}}{g\left(1-r_{\mathrm{B}}\right)}-1\right)^{2}+\frac{1}{8} H^{2}\right]+O\left(H^{4}\right)
$$

It can be verified that, for $U=0$, the expression for $C$ agrees with that given by Tsuji \& Nagata (1973).

The densities of kinetic energy $T$, potential energy $V$, total energy $E$, mass flux (or impulse) $I$, momentum flux (or radiation stress) $S_{x x}$, action $A$, action flux $J$, and energy flux $F$ can be obtained from the following relations:

$$
\begin{gathered}
T=\frac{1}{2} k C L_{i \omega}, \quad V=\frac{1}{2} k C L_{t,}-L, \quad E=k C L_{\omega}-L, \quad I=k L_{\omega}, \\
S_{x x}=-k L_{k}+L, \quad A=L_{\omega}, \quad J=-L_{k}, \quad F=-k C L_{k} .
\end{gathered}
$$

It should be noted that the relation

$$
L_{k}=-\frac{2}{k} L-\frac{1}{2} C L_{\omega}
$$

given by Longuet-Higgins (1975) for surface waves and found to hold for interfacial waves by Holyer (1979), from which follows $S_{x x}=4 T-3 \mathrm{~V}$, no longer holds for $U \neq 0 . \dagger$

The group velocity is not uniquely defined for nonlinear waves. One definition, as used by Whitham $(1974$, p. 514), is the speed of the characteristics for long modulations. This requires calculation of $\partial^{2} L / \partial \omega \partial H$ and $\partial^{2} L / \partial k \partial H$, which can be found via (3.10) from knowledge of the dependence of the integral parameters on $H$. For weakly nonlinear waves, it can be deduced from (3.7) and equation (15.13) of Whitham (1974). Because of the large amount of computation involved, and because of the fact that the concept of group velocity is more relevant to stability and evolution problems, we did not calculate group velocities for finite-amplitude waves here.

\section{Numerical methods}

The most straightforward method for direct numerical computation is to solve (1.1)-(1.3), subject to the conditions (1.4)-(1.6) using collocation techniques. This requires that the discrete-series representation $(2.1)-(2.5)$ satisfies the governing equations and boundary conditions at $N+1$ discrete points located at

$$
x_{i}=\frac{(i-1) \pi}{N} \quad(i=1,2, \ldots, N+1),
$$

$\dagger$ A simple derivation of (3.11) employs the fact from dimensional analysis that

$$
L=g H^{2} \text { function }\left(\frac{\omega^{2}}{g k}, k H, \frac{U k}{\omega}\right) .
$$




\begin{tabular}{|c|c|c|c|c|c|}
\hline$H$ & Holyer & $\begin{array}{c}\text { Physical } \\
N=40\end{array}$ & $B(40) / B(1)$ & $\begin{array}{c}\text { Hodograph } \\
N=40\end{array}$ & $a(40) / a(1)$ \\
\hline $0 \cdot 1$ & $1 \cdot 00104$ & $1 \cdot 0010433$ & $10^{-15}$ & $1 \cdot 0010433$ & $10^{-16}$ \\
\hline $0 \cdot 2$ & $1 \cdot 00417$ & 1.0041725 & $10^{-12}$ & 1.0041725 & $10^{-15}$ \\
\hline $0 \cdot 3$ & 1.00939 & $1 \cdot 0093851$ & $10^{-12}$ & $1 \cdot 0093851$ & $10^{-15}$ \\
\hline $0 \cdot 4$ & $1 \cdot 01668$ & $1 \cdot 0166763$ & $10^{-11}$ & $1 \cdot 0166763$ & $10^{-14}$ \\
\hline $0 \cdot 5$ & $1 \cdot 02604$ & 1.0260381 & $10^{-9}$ & $1 \cdot 0260381$ & $10^{-13}$ \\
\hline $0 \cdot 6$ & $1 \cdot 03746$ & $\mathbf{1} \cdot 0374588$ & $10^{-9}$ & 1.0374588 & $10^{-12}$ \\
\hline 0.7 & $1 \cdot 05092$ & $1 \cdot 0509243$ & $10^{-5}$ & 1.0509243 & $10^{-10}$ \\
\hline $0 \cdot 8$ & $1 \cdot 06643$ & $1 \cdot 0664230$ & $10^{-4}$ & $1 \cdot 0664230$ & $10^{-9}$ \\
\hline 0.9 & 1.0841 & $1 \cdot 0839601$ & $1 \cdot 2$ & $1 \cdot 0839602$ & $10^{-8}$ \\
\hline
\end{tabular}

TABLE 1. Comparison of calculated wave speed for various values of wave height $H$. Left to right: first column - wave height $H$; second column - wave speed $C$ obtained by Holyer (Holyer 1981, private communications); third column - wave speed $C$ obtained with physical space method using 40 modes; fourth column - ratio of last to first Fourier coefficient of velocity potential below for the physical-space method; fifth column - wave speed $C$ obtained with hodograph-space formulation using 40 modes; sixth column - ratio of last to first Fourier coefficients of velocity potential below for the hodograph-space method.

resulting in $3 N+3$ equations for the $3 N+4$ unknowns $A_{1}, \ldots, A_{N}, B_{1}, \ldots, B_{N}$, $B_{1}^{\prime}, \ldots, B_{N}^{\prime}, b, B_{0}, B_{0}^{\prime}$ and $C$. The remaining equation (2.7) is the definition of $H$. These equations were solved by Newton's method, using continuation in $U$ and/or $H$ to give the first guesses. Note that this formulation is essentially the same as Holyer's (1979), the only difference being in the way the coefficients are cast and calculated.

The accuracy of the calculated solutions is checked by (2.11) and is tested by its lack of dependence on $N$.

The method works extremely well for small values of $H$. The limits of reliability depend, of course, on the values of $r$ and $U$. The method must fail if the surface becomes 'overhanging' - when $Y$ is not a single-valued function of $x$. The series (2.1) is expected to converge very slowly, if at all, when the slope of the wave becomes steep, so that the method would fail when the profile is near-vertical. It should be noted, however, that this failure of the collocation method does not necessarily mean that a limiting solution has been reached.

A more serious deficiency in this simple formulation is that the series $(2.2)-(2.5)$, which give the representations for the velocity potentials and stream functions above and below the interface, may fail to converge for some values of $x$ on $Y(x)$ even though the solution may be well-behaved. This happens when the analytic continuation of the lower velocity potential into the upper region develops a singularity lying below the level of the crest, or when the analytic continuation of the upper velocity potential into the lower region develops a singularity lying above the level of the trough. This type of failure of convergence is reflected in the non-decrease for large $n$ of the Fourier coefficients $B_{n}$ and $B_{n}^{\prime}$. An example of this behaviour (for $r=0 \cdot 1, U=0$ ) is given in table 1 , where we have tabulated the ratios of the last to the first Fourier coefficients of the velocity potential below. It can be seen that, for $H \approx 0 \cdot 9$, the Fourier representation clearly does not converge, even though the wave is smooth and the integral properties (such as the wave speed $C$ ) appear not to be strongly affected.

To circumvent this convergence difficulty due to singularities of analytic continuations of the velocity potentials, we adopt a second approach by working in the hodograph plane. In this formulation we express the physical coordinates $x$ and $y$ 
as functions of $\phi$ and $\psi$ below the interface, and $\phi^{\prime}$ and $\psi^{\prime}$ above the interface. A subsidiary complex variable $\xi+i \eta$ is introduced by the definitions

$$
\xi+i \eta=-\frac{1}{C}(\phi+i \psi)=\frac{1}{U-C}\left(\phi^{\prime}+i \psi^{\prime}\right)
$$

In terms of $\xi$ and $\eta$, the physical coordinates $x$ and $y$ below and $x^{\prime}$ and $y^{\prime}$ above can be expressed in truncated series:

$$
\begin{gathered}
x=\xi+\sum_{1}^{N} a_{n} \sin n \xi e^{n \eta}, \quad y=\eta+a_{0}+\sum_{1}^{N} a_{n} \cos n \xi e^{n \eta}, \\
x^{\prime}=\xi+\sum_{1}^{N} a_{n}^{\prime} \sin n \xi e^{-n \eta}, \quad y^{\prime}=\xi+\alpha_{0}^{\prime}-\sum_{1}^{N} a_{n}^{\prime} \cos n \xi e^{-n \eta}
\end{gathered}
$$

It is easily seen by considering the flow at infinity that

$$
a_{0}=\frac{B_{0}}{C}, \quad a_{0}^{\prime}=\frac{B_{0}^{\prime}}{C-U} .
$$

On the interface, which is chosen to be $\eta=0$, the tangential velocities below and above are given by

$$
\begin{gathered}
q=-C\left[\left(1+\sum_{1}^{N} n a_{n} \cos n \xi\right)^{2}+\left(\sum_{1}^{N} n a_{n} \sin n \xi\right)^{2}\right]^{-\frac{1}{2}} \\
q^{\prime}=(U-C)\left[\left(1+\sum_{1}^{N} n a_{n}^{\prime} \cos n \xi\right)^{2}+\left(\sum_{1}^{N} n a_{n}^{\prime} \sin n \xi\right)^{2}\right]^{-\frac{1}{2}}
\end{gathered}
$$

The Bernoulli equation (1.3) provides one set of $N+1$ equations when it is required to be satisfied at $N+1$ points equally distributed from the crest to the trough:

$$
\xi_{i}=\frac{(i-1) \pi}{N} \quad(i=1,2,3, \ldots, N+1) .
$$

The requirement that the curves (4.3) and (4.4) are the same at the interface provides another set of $2 N$ equations. It is convenient to introduce a new variable $s=s(\xi)$ such that $\xi+s$ above corresponds to $\xi-s$ below. In terms of $\xi$ and $s$, the conditions that $x=x^{\prime}$ and $y=y^{\prime}$ at the interface can be expressed as

$$
\begin{gathered}
\xi-s+\sum_{1}^{N} a_{n} \sin n(\xi-s)=\xi+s+\sum_{1}^{N} a_{n}^{\prime} \sin n(\xi+s), \\
a_{0}+\sum_{1}^{N} a_{n} \cos n(\xi-s)=a_{0}^{\prime}-\sum_{1}^{N} a_{n}^{\prime} \cos n(\xi+s) ;
\end{gathered}
$$

again to be satisfied at $N+1$ points given by (4.8), with the auxiliary equation

$$
s(0)=s(\pi)=0 .
$$

Since (4.9) is satisfied identically at the end points owing to (4.11), we have a total of $2 N$ equations from (4.9) and (4.10). This gives us $3 N+1$ equations for the $3 N+3$ unknowns $a_{1}, \ldots, a_{N}, a_{1}^{\prime}, \ldots, a_{N}^{\prime}, a_{0}, a_{0}^{\prime}, C, b, s_{2}, \ldots, s_{N}$, where $s_{i}=s\left(\xi_{i}\right)$. Two further equations are provided by the definition of wave height,

$$
y(0)-y(\pi)=y_{\mathrm{c}}-y_{\mathrm{t}}=H,
$$




\begin{tabular}{cccc}
$H$ & Hodograph & \\
$0 \cdot 1$ & Holyer & $N=100$ & $a(100) / a(1)$ \\
$0 \cdot 2$ & $1 \cdot 00104$ & $1 \cdot 0010433$ & $10^{-16}$ \\
$0 \cdot 3$ & $1 \cdot 00417$ & $1 \cdot 0041725$ & $10^{-15}$ \\
$0 \cdot 4$ & $1 \cdot 00939$ & $1 \cdot 0093851$ & $10^{-14}$ \\
$0 \cdot 5$ & $1 \cdot 01668$ & $1 \cdot 0166763$ & $10^{-13}$ \\
$0 \cdot 6$ & $1 \cdot 02604$ & $1 \cdot 0260381$ & $10^{-13}$ \\
$0 \cdot 7$ & $1 \cdot 03746$ & $1 \cdot 0374588$ & $10^{-13}$ \\
$0 \cdot 8$ & $1 \cdot 05092$ & $1 \cdot 0509243$ & $10^{-13}$ \\
$0 \cdot 9$ & $1 \cdot 06643$ & $1 \cdot 0664230$ & $10^{-12}$ \\
1.0 & $1 \cdot 0841$ & $1 \cdot 0839603$ & $10^{-12}$ \\
$1 \cdot 1$ & $1 \cdot 103$ & $1 \cdot 1035877$ & $10^{-11}$ \\
1.2 & $1 \cdot 125$ & $1 \cdot 125454$ & $10^{-8}$ \\
1.22 & $1 \cdot 148$ & $1 \cdot 149904$ & $10^{-8}$ \\
$1 \cdot 24$ & $1 \cdot 152$ & $1 \cdot 1552$ & $10^{-6}$ \\
1.26 & $1 \cdot 157$ & $1 \cdot 1606$ & $10^{-6}$ \\
1.28 & $1 \cdot 162$ & $1 \cdot 166$ & $10^{-5}$ \\
1.30 & $1 \cdot 167$ & $1 \cdot 172$ & $10^{-5}$ \\
\hline
\end{tabular}

TABLF 2. Comparison of calculated wave speed for various values of wave height $H$. Left to right: first column - wave height $H$; second column - wave speed $C$ obtained by Holyer (Holyer 1981, private communications); third column - wave speed $C$ obtained with hodograph-space formulation using 100 modes; fourth column - ratio of last to first Fourier coefficients of velocity potential below for the hodograph-space method.

and the requirement that the interface has zero mean, $\dagger$

$$
\bar{y}=\int_{0}^{\pi} y(\xi) \frac{d x}{d \xi} d \xi=0 .
$$

Given the values of $H, r$ and $U$, the resulting $3 N+3$ equations are solved by Newton's method. The relative kinetic energy follows from (2.8) using (4.5), and the potential energy is given by

$$
V=\frac{1}{2 \pi}\left(1-r_{\mathrm{B}}\right) \int_{0}^{\pi}\left(\sum_{0}^{N} a_{n} \cos n \xi\right)^{2}\left(1+\sum_{1}^{N} n a_{n} \cos n \xi\right) d \xi
$$

Accuracy is again tested by the independence of the results on the value of $N$. It can be seen from table 1 that the hodograph method does not suffer from the convergence failure of the physical formulation. In table 2 , we compare the results for the phase speed using 100 modes with those of Holyer (1979) for $r=0 \cdot 1, U=0$, and $H$ up to $1 \cdot 3$. It is interesting to note that good agreement between the two results for phase speed is achieved up to $H=0.9$, approximately the same value of $H$ where the $N=40$ calculations also yield agreeable and acceptable results (compare table 1 ). For $H \geqslant 1.0$, however, disagreement to the fourth significant figure is seen. For $H \geqslant 1 \cdot 2$, the disagreement is to the third significant figure. Our calculations are monitored by the ratio of the last Fourier coefficient to the first, shown in the last column of table 2 , which should be a reasonable indication of the magnitude of error in our results. We are hesitant at this point, however, to attribute the disagreement to inaccuracies in the Padé approximants (when the physical-plane formulation is

$\dagger$ In practice, we took $a_{0}=0$, calculated $\bar{y}$, and made the substitutions $a_{0}=-\bar{y}, a_{0}^{\prime}=a_{0}^{\prime}-\bar{y}$, $b=b+\left(1-r_{\mathrm{B}}\right) \bar{y}$. 


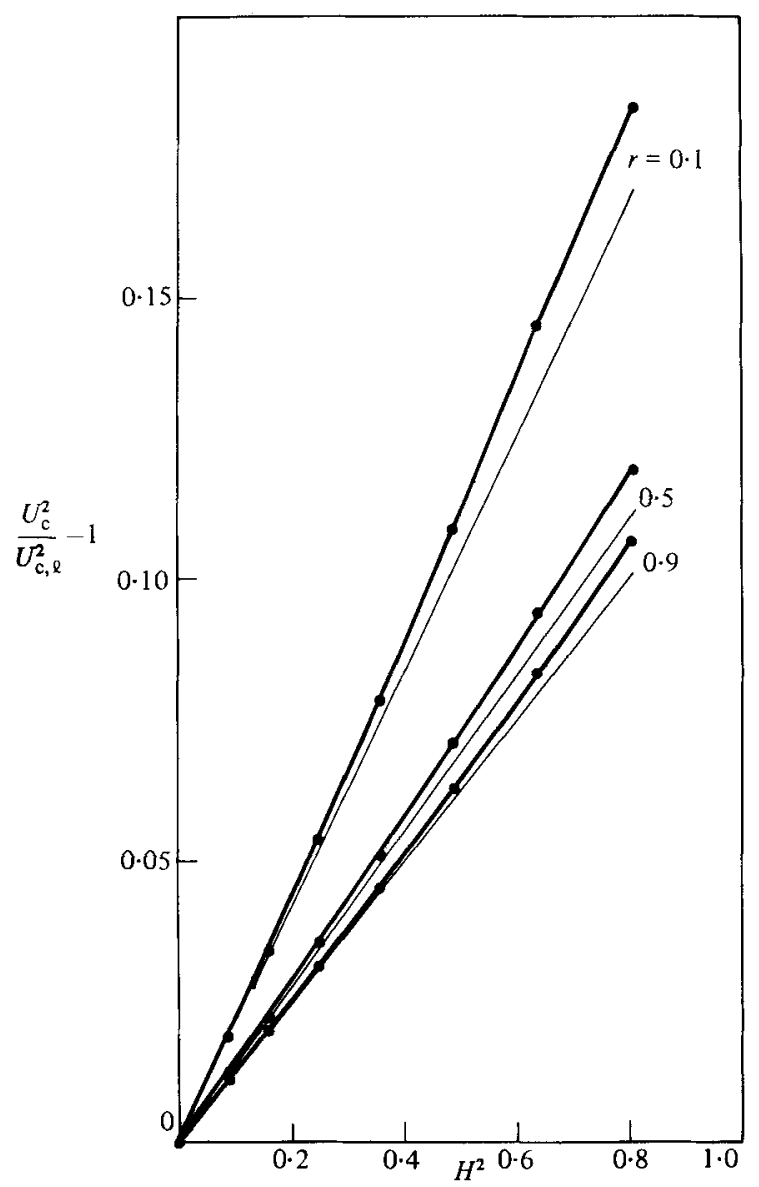

Figure 2. Critical values of current $U=U_{\mathrm{c}}$ as a function of wave height $H$ for various density ratios $r$. Plot of $U_{\mathrm{c}}^{2} / U_{\mathrm{c}, \ell}^{2}-1$ against $H^{2}$. Solid circles represent numerical results obtained with the hodograph-space method using 40 modes. Thin lines represent weakly nonlinear approximations given by (5.3).

non-convergent), since our method is also not suitable for very steep waves. We think that the vortex-sheet methods are probably more efficient, because they allow the concentration of points at regions of large curvature (see e.g. Chen \& Saffman 1980b), and should therefore be employed to verify the steep-wave results to a larger number of significant figures.

\section{Results}

It can be seen from the dispersion relation (3.9) that, for linear waves $(H \rightarrow 0)$ and given values of density ratio $r$ and current velocity $U$, there are two solutions corresponding to the two roots of the quadratic equation for $C$ in terms of $r$ and $U$. We denote these two solutions by $C_{+}$and $C_{-}$, where $C_{+} \geqslant C_{-}$. For the linear case, steady solutions cease to exist when $U$ exceeds a critical value $U_{\mathrm{c} \ell}$ (the second subscript $\ell$ standing for the linear case) given by

$$
U_{\mathrm{c} \ell}=\left[(1+r)\left(1-r_{\mathrm{B}}\right) / r\right]^{\frac{1}{2}}
$$



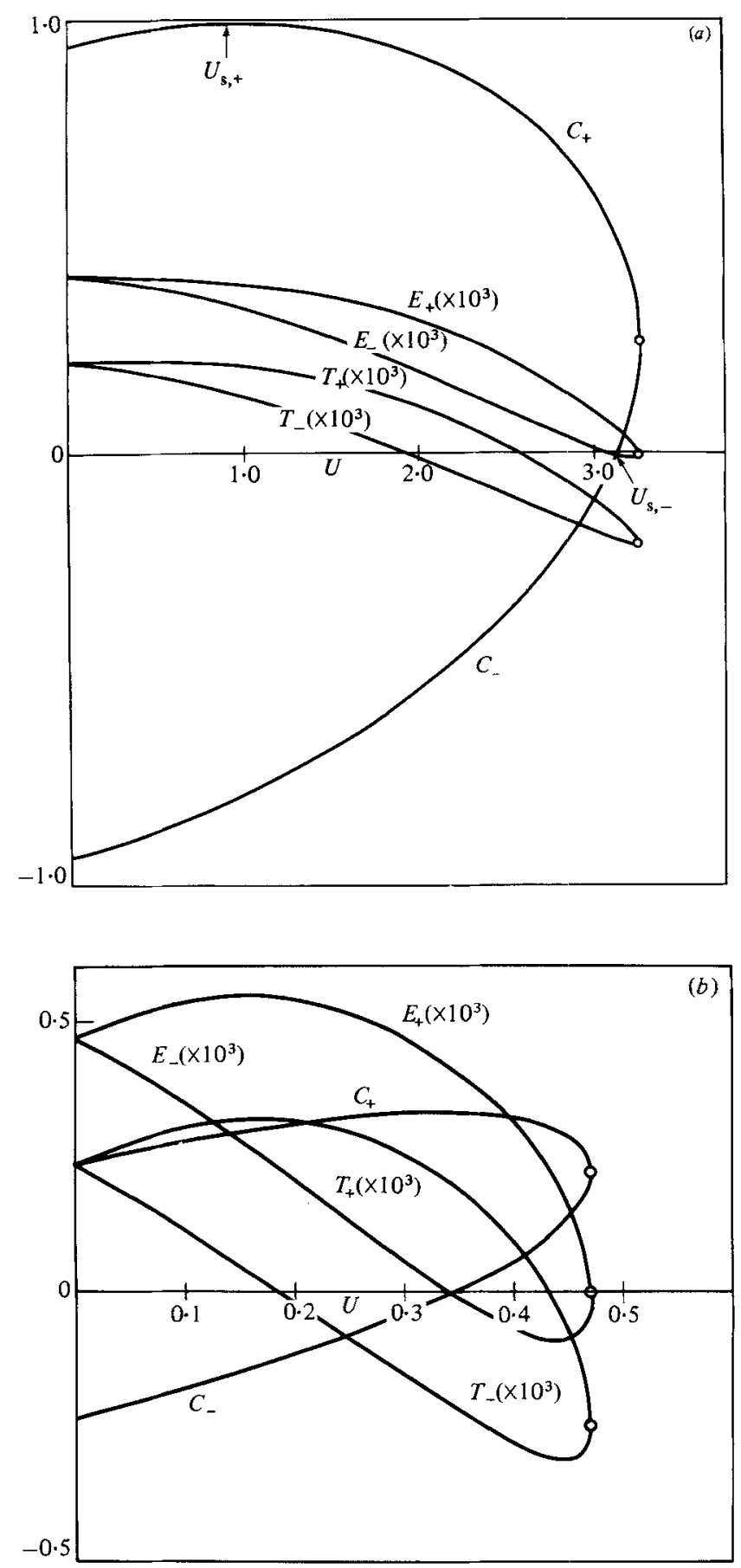

Figure $3(a, b)$. For caption see facing page. 

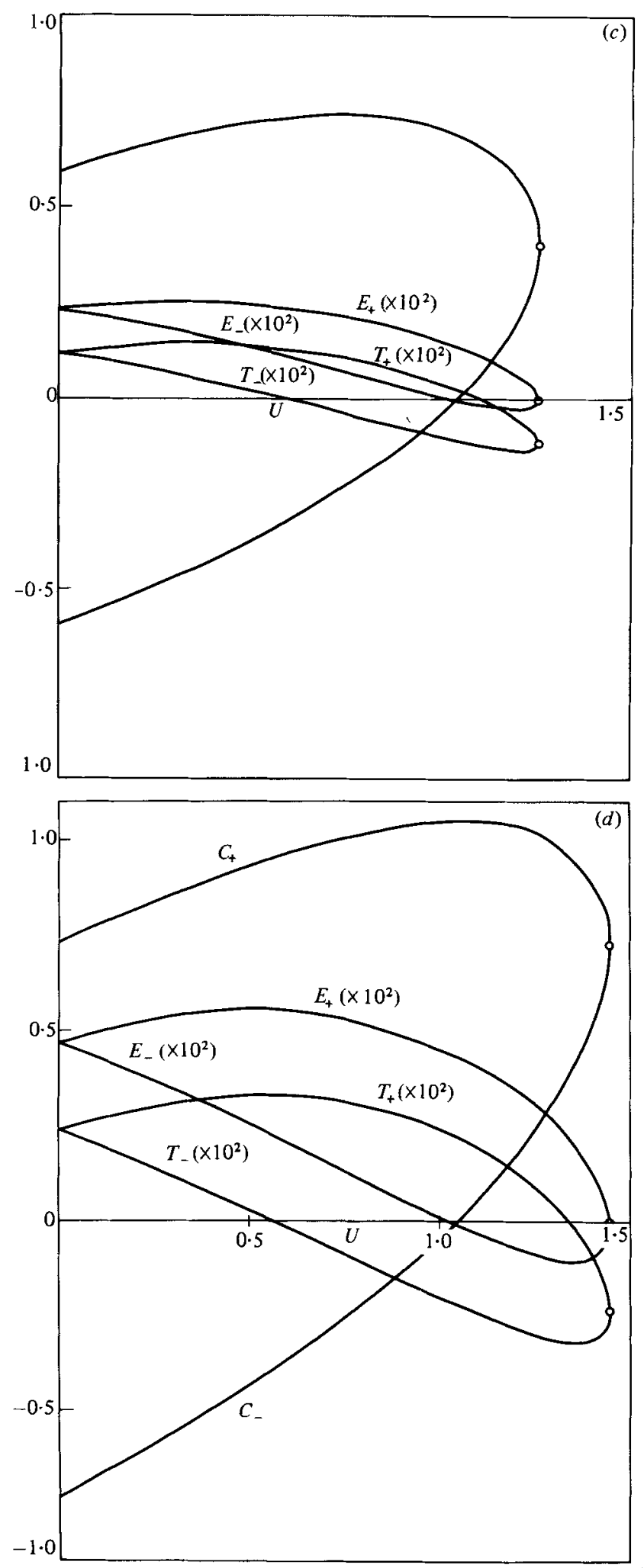

Figure 3. Integral properties of interfacial waves as functions of current velocity $U$ for a fixed wave height $H(H=0 \cdot 6)$. Plots of wave speed $C$, kinetic energy $T$ and total energy $E$ for the + and - branches against current velocity $U \cdot(a) r=0.1$ (points labelled $U_{\mathrm{s}+}$ and $U_{\mathrm{s}-}$ are the sharpest waves for each branch, see discussion on figure 4$) ;(b) 0.5 ;(c) 0.9 ;(d)$ Boussinesq $\left(r=1.0\right.$ and $\left.r_{\mathrm{B}}=0\right)$. 
with equal wave speeds

$$
C_{+}=C_{-}=\frac{r U_{\mathrm{e} \ell}}{1+r}
$$

For finite-amplitude waves $(H \neq 0)$, these two solutions continue into two families of solutions marked by wave speeds $C_{+}(H, r, U)$ and $C_{-}(H, r, U)$. For any given values of $H$ and $r$ there will again be a critical current $U_{c}$, characterized by $C_{+}\left(H, r, U_{\mathrm{c}}\right)=C_{-}\left(H, r, U_{\mathrm{c}}\right)$, beyond which steady solutions no longer exist. It is interesting to note that from the weakly nonlinear approximation, as well as from numerical results to be reported below, this critical current velocity is increased for increasing $H$ for a given value of $r$. The value of $U_{e}$, correct to second order in $H$, can be obtained by equating the two roots of (3.9),

with

$$
\begin{gathered}
U_{\mathrm{c} 2}=U_{\mathrm{c} \ell}\left[1+\frac{H^{2}\left(1+r^{2}\right)}{4(1+r)^{2}}\right]^{\frac{1}{2}}, \\
C_{+, 2}=C_{-, 2}=\frac{r U_{\mathrm{c} 2}}{1+r},
\end{gathered}
$$

where the second subseript 2 stands for second-order approximation.

The result that increasing $H$ increases $U_{\mathrm{c}}$ is interesting, since it can be vicwed as a stabilization of parallel flows by waves. This is because for given values of $r$ and $U$ steady interfacial configurations exist only if there are waves with heights greater than some minimum values. The significance of this observation must await stability considerations of the steady interfacial waves, which will be reported in a fortheoming paper.

We now present the results of numerical calculations of $U_{\mathrm{c}}$ for three values of density ratio $(r=0.1,0.5,0.9)$, each for a range of wave height $0 \leqslant H \leqslant 0.81$. These are shown in figure 2 . The numerical results compare well with the weakly nonlinear results of (5.3), indicating that we are still working in the range of small-to-moderate wave amplitudes. The agreement improves with increasing $r$ owing to the fact that the geometrically limiting wave height for $U=0$ increases with increasing $r$ from $H=0.892$ for $r=0$ (Stokes surface limiting wave) to $H=2 \cdot 25$ for the Boussinesq wave (Holyer 1979). The Boussinesq-wave solution is obtained by setting $r=1$ and $r_{\mathbf{B}}=0$; the results for the Boussinesq case are practically indistinguishable from the $r=0.9$ case for the values shown in figure 2 and are therefore not exhibited.

In figure 3 we show the integral properties for density ratios $r=0 \cdot 1,0 \cdot 5,0 \cdot 9,1 \cdot 0$ (Boussinesq), as functions of $U$ for a chosen value of $H(H=0 \cdot 6)$. These results were obtained using $\mathbf{4 0}$ modes in the hodograph formulation. The last Fourier coefficients were $O\left(10^{-10}\right)$. Increasing the number of modes to 80 and 100 did not alter the results to six significant figures, and there was full agreement with results obtained with the physical-space formulation using the same number of modes (see e.g. table 1 for the $U=0, r=0.1$ ease). We have plotted, for each value of $r$, properties associated with the two branches of solutions characterized by $C_{+}$and $C_{-}$respectively. In particular, we show the wave speeds, the kinetic energies $T_{+}$and $T_{-}$, the total energies $E_{+}$and $E_{-}$, as functions of current velocity $U$. Notice that both branches of solutions meet when $U=U_{\mathrm{c}}$. Also note that for each value of $r$ there is a region of $U$ near $U=U_{\mathrm{c}}$ where the state energy of the wave system is in fact less than that for parallel flow. Whether or not this means that a state with strong current and large waves is preferred over parallel flow must await a stability analysis.

In figure 4 we present the wave profiles for selected values of $U$ from $U=0$ to $U=U_{\mathrm{c}}$ for $H=0 \cdot 6$ and $r=0 \cdot 1$. We first note that the $C_{+}$waves are peaked at the 


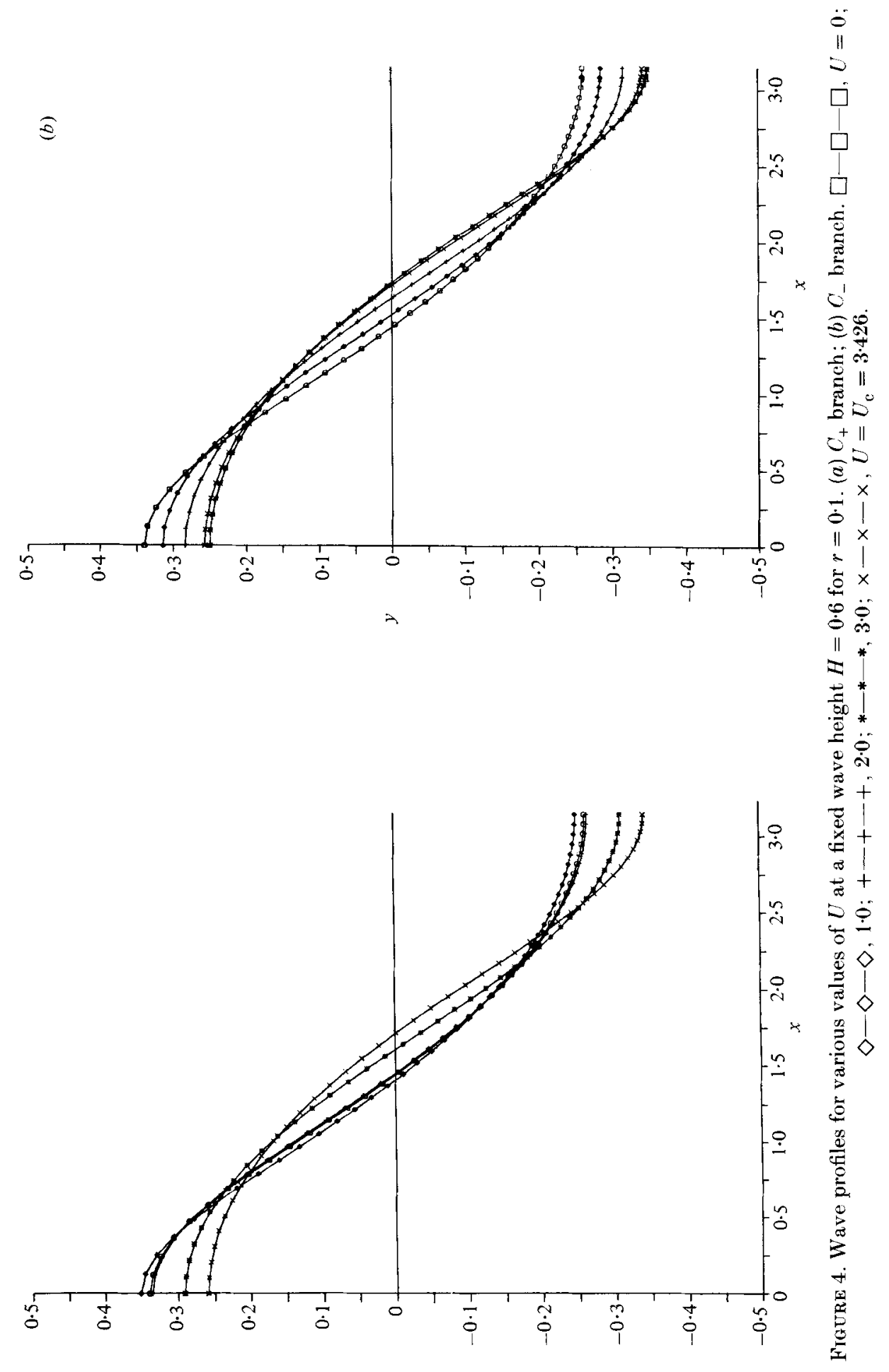




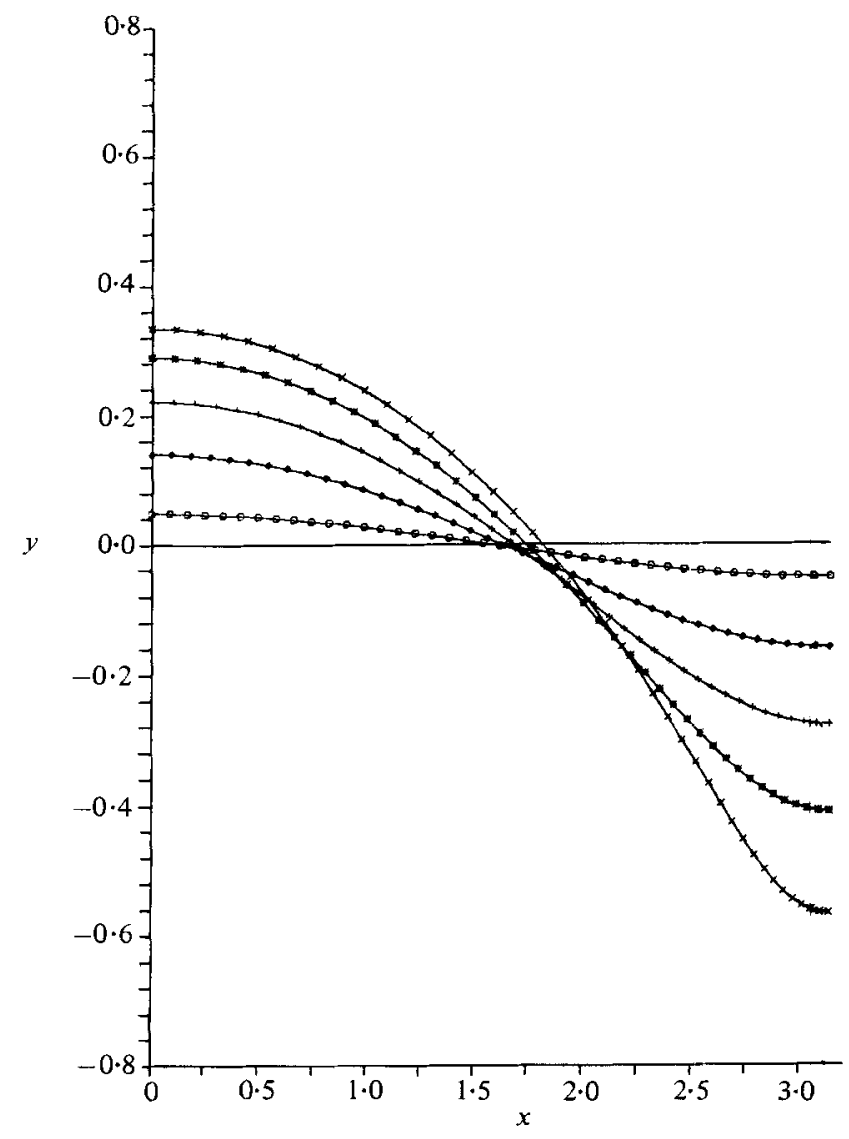

Figure 5. Wave profiles of current-limited waves $\left(U=U_{\mathrm{c}}\right)$ for $r=0.1$ and various values of $H$. $\square-\square-\square, H=0.1 ; \diamond-\diamond-\diamond, 0.3 ;+-+-+, 0.5 ; *-*-*, 0.7 ; \times-\times-\times, 0.9$.

crest, whereas the $C_{-}$waves are peaked at the trough. Another feature is outstanding: the sharpest wave (i.e. the wave with the largest crest or trough curvature) occurs for a value of $U$ that lies inside the range $\left(0, U_{\mathbf{c}}\right)$. Denoting such a value of $U$ by $U_{\mathrm{s}+}$ for the $C_{+}$wave, and $U_{\mathbf{s}-}$ for the $C_{-}$wave, we find that $U_{\mathbf{s}+}$ is always less than $U_{\mathbf{s}-}$. This qualitative behaviour is present for all values of $r$, but becomes less pronounced as $r$ increases. It should be noticed that Boussinesq waves with $U \neq 0$ are not symmetrical about the mean (as would be the case if $U=0$ ). One speculation, supported by the trend of the results, is that the wave associated with $U_{\mathrm{s}+}$ or $U_{\mathrm{s}-}$ will become increasingly sharp at the erest or the trough as $H$ increases, and a geometrically limiting wave, one for each branch of solutions, will be reached. The geometrical limitation is presumably near the crest for the $C_{+}$waves and near the trough for the $C_{-}$waves, and except for a special case discussed in $\S 6$, where a sharp corner is formed at the crest or trough, is probably associated with the surface crossing itself so that the solutions become unphysical. $\dagger$

In figure 5, we plot the current-limited waves $\left(U=U_{\mathrm{c}}\right)$ for $t=0 \cdot 1$ and $H=0 \cdot 1$,

† Holyer (1979) proposed the criterion that waves are limited by the occurrence of a vertical tangent in the profiles, which must take place prior to self-crossing. We fail to find any dynamical or kinematical reasons to support her choice, which is certainly not applicable to surface gravity-capillary waves (Chen \& Saffman 1980a). 
$0.3,0.5,0.7$ and 0.9 . As $H$ increases, the waves become increasingly sharp at the trough. This tendency is most noticeable for $r=0.1$, but is present for all $r$, weakening, however, as $r \rightarrow 1$. For Boussinesq waves, where $r=1$ and $r_{\mathrm{B}}=0$, the limiting wave is symmetric about the mean, and this tendency must vanish. This is consistent with the above observation that $U_{\mathrm{s}+}$ is less than $U_{\mathrm{s}-}$, since it implies that the $U=U_{\mathrm{c}}$ solution is 'closer' to the $U=U_{\mathrm{s}-}$ wave, which has a sharp trough.

\section{A special class of solutions}

It is interesting to note that a special class of solutions exist, which are simple transformations of the Stokes (1847) surface permanent-wave solutions (calculated numerically for values of $H$ up to the limiting height by Schwartz 1974; Cokelet 1977 , among others).

For each value of $r$, these solutions exist for the $C_{+}$case when

$$
C_{+}=U=\left(1-r_{\mathrm{B}}\right)^{\frac{1}{2}} C_{\mathrm{s}}(H),
$$

where $C_{\mathrm{s}}(H)$ is the wave speed of the Stokes waves for the given wave height $H$. Since $C=U$, the upper fluid is stagnant in the wave-fixed coordinates. The shape of the surface is identical with that of the Stokes waves of the same height, and the velocities and wave speed are those of the Stokes wave multiplied by the factor $\left(1-r_{B}\right)^{\frac{1}{2}}$. It is easily verified that this gives an exact solution of (1.1)-(1.3).

For the $C_{-}$branch, special solutions exist with

$$
C_{-}=0, \quad U=\left[\frac{1-r_{\mathrm{B}}}{r}\right]^{\frac{1}{2}} C_{\mathrm{s}}(H) .
$$

In this case, the lower fluid is stagnant, and the velocities above are those of a Stokes wave with negative gravity multiplied by the factor $\left[\left(1-r_{\mathrm{B}}\right) / r\right]^{\frac{1}{2}}$. The profiles are inverted or upside-down Stokes waves.

Obviously, these special solutions possess geometrical limits when $H \approx 0.892$ (see e.g. Stokes 1880; Michell 1893; Schwartz 1974; Cokelet 1977), where the waves have a corner at the crest (or the trough, as the case may be) with an interior angle of $120^{\circ}$.

\section{Remarks on the existence of steady solutions}

In figure 6 , we present a plot in the $(r, U)$-plane when $H=0.892$. The solid line shows the critical value of $U$ according to the second-order approximation given by (5.3) $\left(U=U_{\mathrm{c} 2}\right)$ for each density ratio $r$ above which steady solutions do not exist (the 'dynamical limit' discussed in $\$ 1$ ). Steady solutions exist for all regions below this line. The lower dashed curve (labelled + ) represents solutions given by $(6.1)$ with $H=0.892$, which possess sharp corners at their crests. The upper dashed curve represents solutions given by (6.2) (again with $H=0.892$ ), which possess sharp corners at their troughs (the inverted limiting Stokes waves). It is expected that for larger values of $H$ regions around the two dashed curves may arise within which steady solutions do not exist because of 'geometric limitations' as discussed in $\S 1$.

In figure 7 , we sketch a possible domain diagram in the $(U, H)$-plane for the existence of steady solutions for a fixed density ratio $(r=0 \cdot 1)$. The solid lines represent actual solutions obtained either by numerical means (the heavy line on top represents the dynamical-limit curve obtained from numerical calculations) or analytical transformations (the two solid thin curves representing the Stokes-like 


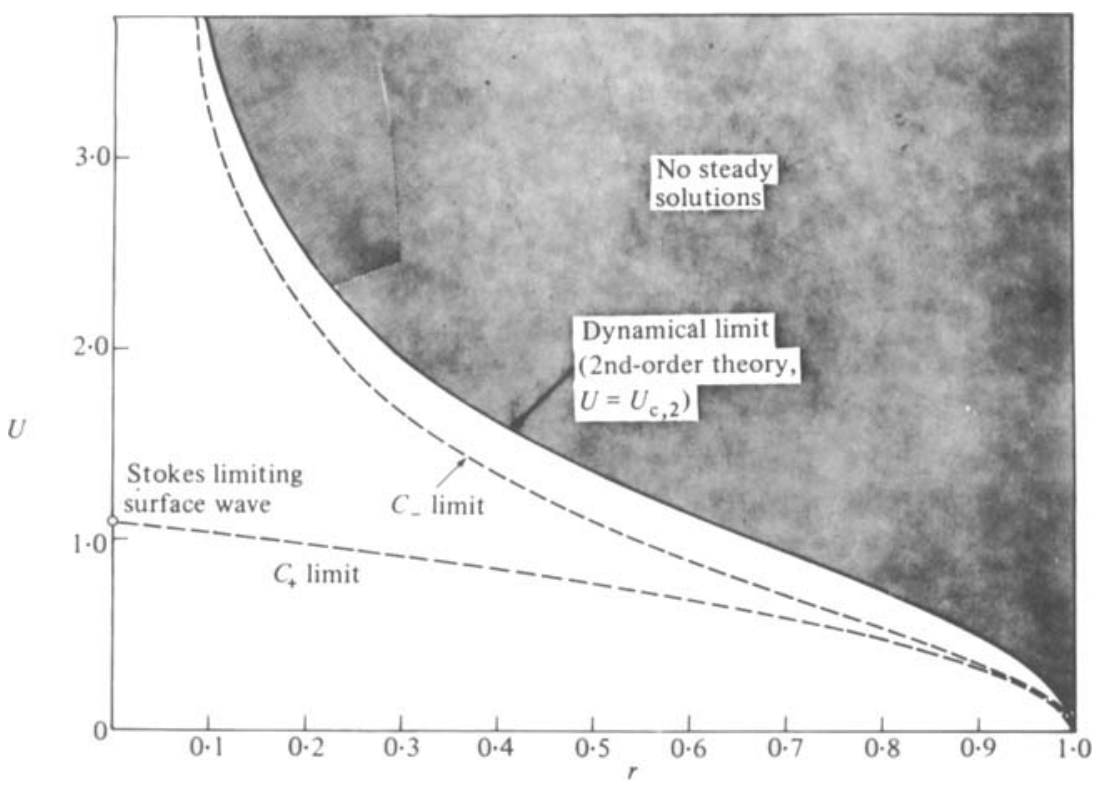

Figtre 6. Dynamical and geometrical limitations on the wave system at $H \approx 0.892$.

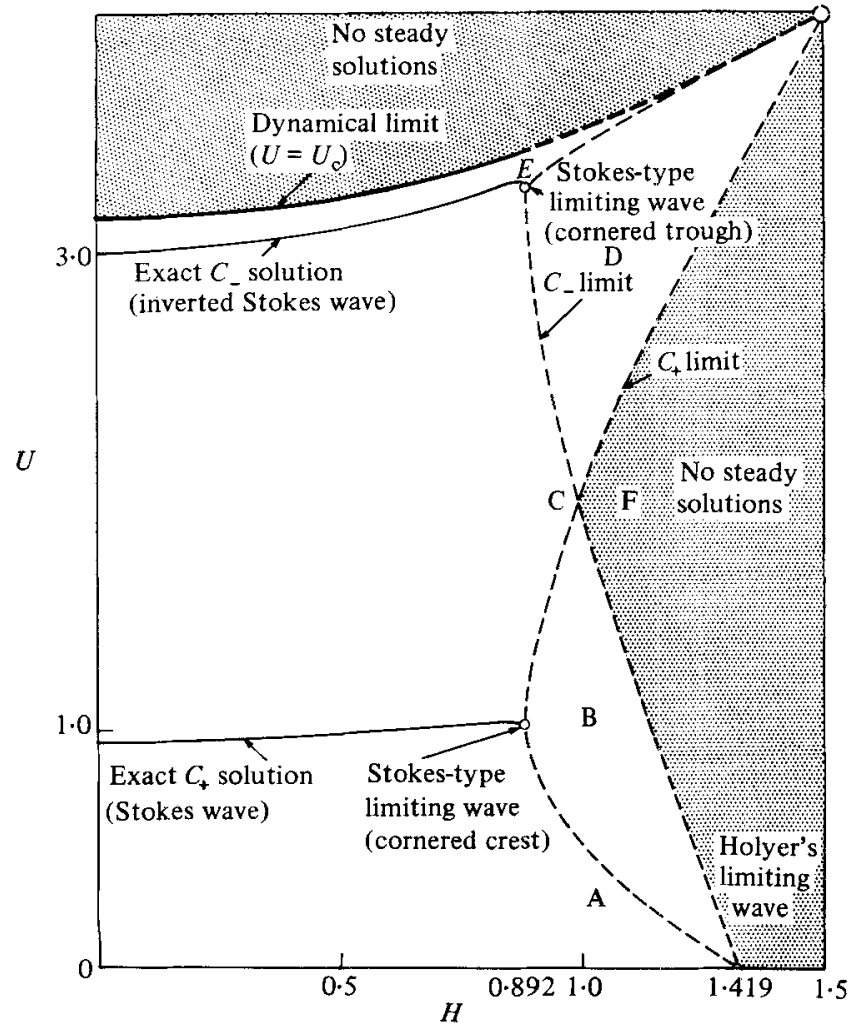

Figure 7. Sketch of solution domain for $r=0 \cdot 1$. 
solutions for the $C_{+}$and $C_{-}$branches). The three circles represent the two Stokes limiting waves, one for each branch of solutions, and the limiting wave reported by Holyer for $U=0$ (see, however, the discussion and footnote on p. 472 regarding Holyer's criterion for a limiting wave). The dashed lines are conjectured extensions from the dynamical-limit curve and the two geometrical-limit points. We expect no solutions to exist in the region lying above the dynamical line and its conjectured extension. We expect no $C_{+}$wave solutions to exist lying to the right of the conjectured geometrical-limit line marked ' $C_{+}$limit', and no $C_{-}$wave solutions to exist lying to the right of the conjectured geometrical-limit line marked ' $C_{-}$limit'. Conversely, we expect solutions to exist everywhere else.

We have mentioned earlier that our methods of calculation are not adequate to handle sharp waves. However, we do possess numerical evidence in support of our conjectures: (i) we found both $C_{+}$and $C_{-}$solutions in parts of regions marked $\mathrm{A}, \mathrm{C}$ and $\mathrm{E}$ in the diagram; (ii) we found $C_{+}$wave solutions, but no $C_{-}$wave solutions, in parts of the region $\mathrm{D}$; (iii) we found $C_{-}$wave solutions, but no $C_{+}$wave solutions, in parts of the region $B$; (iv) we found no solutions in region $F$, nor in the region above the dynamical-limit line. It should be emphasized once again, however, that our methods are not suitable for sharp waves, so that our failure to obtain solutions (typically, the solution cannot be continued since Newton's method diverged, even for very small increments) is not conclusive, but only suggestive, on the question of non-existence.

This work was partially supported by the National Science Foundation and the Office of Naval Research.

\section{Appendix}

In wave-fixed coordinates, the Bernoulli equations below and above can be written as

$$
\begin{gathered}
p+\frac{1}{2} q^{2}+y+b=0, \\
p^{\prime}+\frac{1}{2} r q^{\prime 2}+r_{\mathrm{B}} y=0 .
\end{gathered}
$$

Consider the static equilibrium between $y= \pm D$ (where $D \gg 1$ ) of the fluid in one wavelength. With negligible error, we omit the dependence of $p$ on $x$ and obtain

$$
p(-D)-p^{\prime}(D)=\left(1+r_{\mathrm{B}}\right) D
$$

where we have used the fact that the mean elevation of the interface $\bar{y}=0$. Since $q(-D)=-C, q^{\prime}(D)=U-C$, it follows from (A 1) to (A 3) that

$$
\frac{1}{2} C^{2}+b=\frac{1}{2} r(C-U)^{2} .
$$

This is (2.11). Note that it remains true if surface tension is acting, because the net surface-tension force across the interface vanishes.

The kinetic-energy density relative to the undisturbed motion is

$$
\left.T=\frac{1}{4 \pi} \int_{0}^{2 \pi} \int_{-D}^{Y}\left\{(u+C)^{2}+v^{2}\right\} d y d x+\frac{1}{4 \pi} r \int_{0}^{2 \pi} \int_{Y}^{D}\left\{u^{\prime}+C\right)^{2}+v^{\prime 2}\right\} d y d x-\frac{1}{2} r U^{2} D,
$$

where $u, v$ are velocity components in the $x$ - and $y$-directions respectively $\left(u^{2}+v^{2}=q^{2}\right.$, $\left.u^{\prime 2}+v^{\prime 2}=q^{\prime 2}\right)$. Expanding the expression and applying Green's theorem with the fact that $\psi=\psi^{\prime}=0$ on $y=Y(x)$, and $\psi(-D) \sim C D+B_{0}, \psi^{\prime}(D) \sim(U-C) D+B_{0}^{\prime}$, we obtain

$$
T=-\frac{1}{2} C B_{0}+\frac{1}{2} r(C+U) B_{0}^{\prime}
$$


The momentum flux relative to the undisturbed motion is

$$
\begin{aligned}
S_{x x}=\frac{1}{2 \pi} \int_{0}^{2 \pi} \int_{-D}^{Y}\left\{p+(u+C)^{2}\right\} d y d x & +\frac{1}{2 \pi} \int_{0}^{2 \pi} \int_{Y}^{D}\left\{p^{\prime}+\left(u^{\prime}+C\right)^{2}\right\} d y d x \\
& +\int_{-D}^{0} y d y+r_{\mathrm{B}} \int_{0}^{D} y d y / r \int_{0}^{D}\left(C^{2}-2 U C\right) d y
\end{aligned}
$$

Substituting for $p$ and $q$ from the Bernoulli equations (A 1) and (A 2), and using the identity

$$
u^{2}-v^{2} \equiv \frac{\partial}{\partial y}\left\{y\left(u^{2}-v^{2}\right)\right\}-2 \frac{\partial}{\partial x}(y u v),
$$

we integrate by parts to obtain, after some algebra,

$$
\begin{aligned}
S_{x x}=-2 C B_{0}+2 r C B_{0}^{\prime}-\frac{1-r_{\mathrm{B}}}{4 \pi} \int_{0}^{2 \pi} & Y^{2}(x) d x \\
& +\left.\frac{1}{4 \pi} \int_{0}^{2 \pi} Y\left\{u^{2}+v^{2}-r\left(u^{\prime 2}+v^{\prime 2}\right)\right\}\right|_{y=Y(x)} d x .
\end{aligned}
$$

In arriving at the form in (A 9), we have also used the result that

$$
\frac{d Y(x)}{d x}=\frac{v}{u}=\frac{v^{\prime}}{u^{\prime}} \quad \text { on } \quad y=Y(x) .
$$

We now substitute (A 1) and (A 2) into (A 9), use the average equality of $p$ and $p^{\prime}$ on the interface, and apply the expression for $T$ from (A 6) to get

$$
S_{x x}=4 T-3 V-2 r U B_{0}^{\prime},
$$

where the potential energy $V$ is

$$
V=\frac{1-r_{\mathrm{B}}}{4 \pi} \int_{0}^{2 \pi} Y^{2}(x) d x
$$

\section{REFERENCES}

Chen, B. \& Saffman, P. G. $1980 a$ Steady capillary-gravity waves on deep water. II. Numerical results for finite amplitude. Stud. Appl. Math. 61, 95.

Chen, B. \& Saffman, P. G. $1980 b$ Numerical evidence for the existence of new types of gravity waves of permanent form on deep water. Stud. Appl. Math. 62, 1.

Cokelet, F. D. 1977 Steep gravity waves in water of arbitrary uniform depth. Phil. Trans. R. Soc. Lond. A 286, 183.

CRAPPER, G. D. 1957 An exact solution for progressive capillary waves of arbitrary amplitude. J. Fluid Mech. 2, 532.

Holyer, J. Y. 1979 Large amplitude progressive interfacial waves. J. Fluid Mech. 93, 433.

LAмB, H. 1932 Hydrodynamics, 6th edn. Cambridge University Press.

Longuet-Higains, M. S. 1975 Integral properties of periodic gravity waves of finite amplitude. Proc. R. Soc. Lond. A 342, 157.

Michell, J. H. 1893 The highest waves in water. Phil. Mag. $36(5), 430$.

Schwartz, L. W. 1974 Computer extension and analytic continuation of Stokes expansion for gravity waves. J. Fluid Mech. 62, 553 .

Stokes, G. G. 1847 On the theory of oscillatory waves. Trans. Camb. Phil. Soc. $8,441$.

STokes, G. G. 1880 Supplement to a paper on the theory of oscillatory waves. Mathematical and Physical Papers of G. G. Stokes, vol. 1, p. 314. Cambridge University Press.

Tsuji, Y. \& Nagata, Y. 1973 Stokes expansion of internal deep water waves to the fifth order. J. Oceanogr. Soc. Japan 29, 61.

Whiтhaм, G. B. 1974 Linear and Non-linear Waves. Wiley-Interscience. 